\title{
Data Mining and Risk Early-warning Analysis of Social Security
}

\author{
Wang Xu \\ Policing Administration Department, Liaoning Police College, Dalian Liaoning China
}

Keywords: public security, data mining, risk, risk early-warning.

\begin{abstract}
It is a hot and difficult problem to predict the risk of community security in a region at present. It is simple and feasible for the grass-roots supervision department to evaluate the unqualified rate of the community safety in the jurisdiction. However, this method cannot well reflect the changing trend of risk factors in some communities in a certain area. To compensate for this shortcoming, this paper attempts to build a community risk coefficient evaluation system. From the perspective of evaluation objects and supervision, the community risk factor evaluation system analyzes and discusses the connotation of community security, the types of the hazard factors in the community and the relationship between the impact of the community security and the community security. On this basis, the construction principle, index system and calculation method of community risk coefficient are put forward, and the community risk coefficient is constructed, which is simple, objective and maneuverable; based on community risk coefficient, it combines the prediction function of BP artificial neural network model to the data trend, and applies the community risk coefficient to various societies successfully. The area, the safety trend prediction and early warning of all kinds of parameters are to be tested, to provide scientific theoretical basis for the grass-roots community safety supervision.
\end{abstract}

\section{Introduction}

In recent years, with the steady and continuous development of computer hardware technology, more and more powerful data collection devices and storage media have entered the market for capacity and functionality, and have also greatly promoted the development of databases. A large amount of information and data are stored in the database. How to turn these massive data into valuable information requires effective methods. Data mining technology is such an effective way to eliminate the gap between data and knowledge and transform data into knowledge. As the name implies, big data mining is the mining of potential and implicit information from huge data, extracting information implied from large, incomplete, disturbing, obscure, and random data. If we can find obvious rules and trends in mining from a large number of data sources, the rules and trends excavated can be summarized, understood and designed by people. It is a very effective big data mining method.

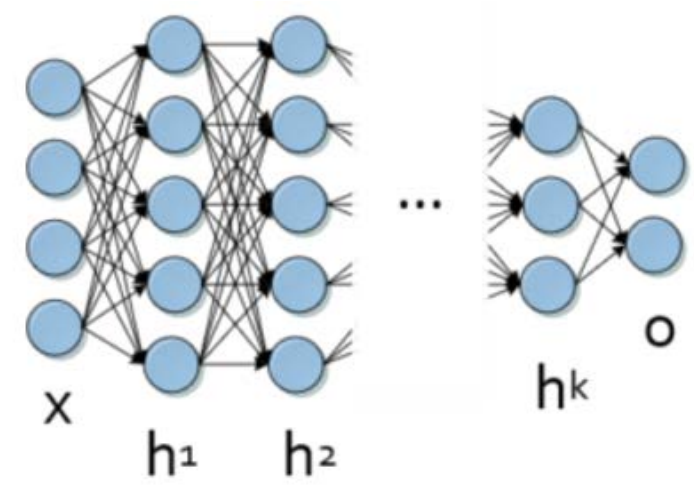

Fig. 1 Network model

Data mining has a variety of classification methods [1]. According to the mining object division, 
there are relational databases, object-oriented databases, spatial databases, temporal databases, text databases, multimedia databases, heterogeneous databases, legacy databases, and so on; According to the excavation task, it can be divided into classification or warning model discovery, data summary, clustering, association rule discovery, sequence pattern discovery, dependency relationship or dependency model discovery, anomaly and trend discovery, etc. [2-4]. According to the mining method, it can be divided into machine learning method, statistical method, neural network method and database method.

\section{Evaluation Object}

\subsection{The significance of community safety assessment.}

Community safety includes community safety, quality and safety, and sustainable safety, while community quality and safety and sustainable safety are the ultimate goals of community safety. Without quality and safety, quantity security is also ineffective, and sustainable security cannot guarantee it. For related departments, in order to strengthen community safety supervision, it is necessary to correctly evaluate the safety status of various communities in the region. Based on a thorough investigation of the status quo of community supervision in the region, the author determines the object of community security risk assessment in the paper.

\subsection{Community safety evaluation object.}

The community risk warning system targets the factors that may be harmful to consumers' health in the community. This requires that the evaluation of community safety should serve the supervision of the supervisory authorities on various influencing factors, that is, based on the judgment conclusions that the hazardous factors in the community do not meet the safety standards, it can be found that the hazardous substances in the community do not meet the safety requirements. The key factors required by the standard, so that the regulatory authorities take targeted supervision. In the study of the comprehensive assessment indicators for community safety, the diversity of communities, the diversity of hazardous substances, and the differences in the hazards of various hazardous substances to human health need to be considered.

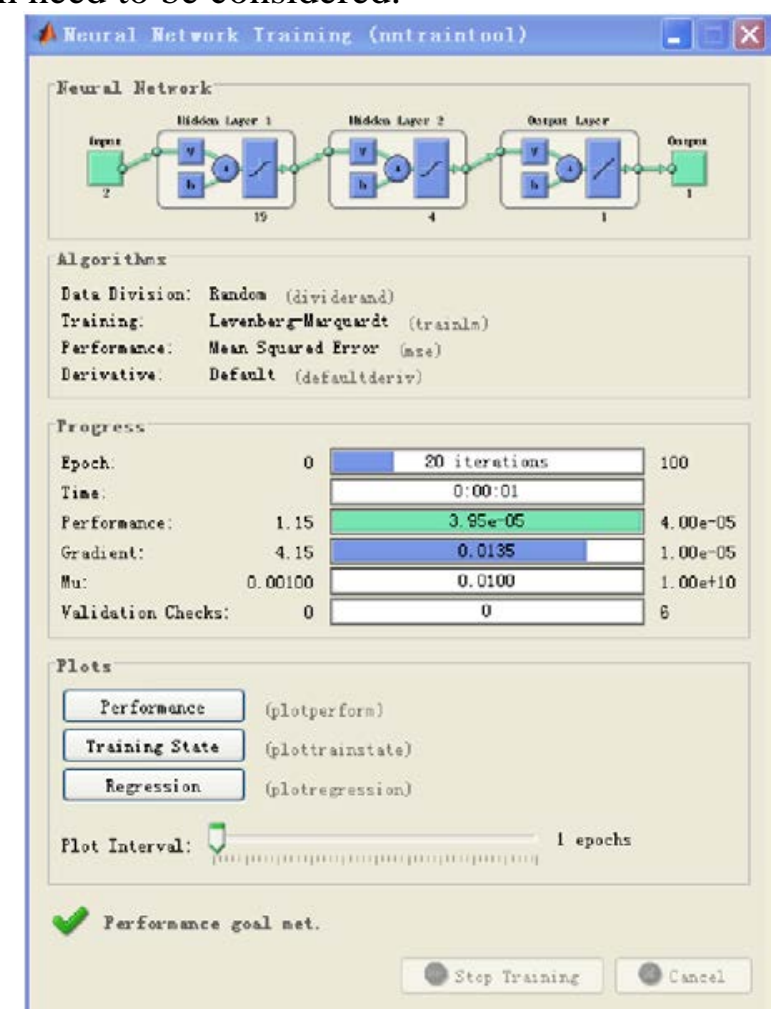

Fig. 2 Network structure and operation process 
The comprehensive evaluation of community security risk involves many indicators and large amount of data, and the same projects have different requirements in different communities. Therefore, the evaluation process should take into account the characteristics of community diversity, the diversity of public security factors and the different degree of harm of public security factors. Therefore, it is necessary to design a quantitative indicator that can reflect the existence of various community security hazards in the community, and to use a mathematical model or a numerical value to represent the recent state of the community security.

\section{Design and Analysis of Risk Factor}

In our country, the vast area, the community category, and even the existence of some public security factors are quite different. Therefore, in the actual daily community safety supervision, especially in the supervision departments of the grass-roots units, the daily supervision and inspection can not be used to detect all the risk factors in the community. It is only for certain high-risk, concerned risk factors for the purpose of sampling, that in the actual risk factor detection data, the detection community does not contain all the corresponding risk factors, so the design evaluation method should be able to make use of the existing community safety of the regulatory department. All the test data were evaluated.

$$
\begin{aligned}
X_{i j} & =\frac{\sum_{n_{i j}} C_{i j}}{n_{i j}} \\
X_{i} & =\left\{\begin{array}{lcc}
0 & \sum_{j}^{J} A_{i j}=0 & \mathrm{j}=1,2, \ldots . ., \mathrm{J} \\
1 & \sum_{j}^{J} A_{i j}=1 & \mathrm{j}=1,2, \ldots \ldots, \mathrm{J}
\end{array}\right. \\
\mathrm{C}_{i j} & =\left\{\begin{array}{lc}
1-\frac{T_{i j}}{\text { Min }_{i j}} & 0<T_{i j}<\operatorname{Min}_{i j} \\
1-\left(\frac{T_{i j}}{\operatorname{Max}_{i j}}\right)^{-1} & T_{i j}>\operatorname{Max}_{i j}>0
\end{array}\right.
\end{aligned}
$$

Community safety risk warning system is an important means of community safety production supervision and improvement of community production level. In many parts of China, under the leadership of the government, a relatively complete community safety risk warning system has been established, and it can better serve the market economy development.

Regulatory personnel through logging in system, inspection standards, inspection information, regulatory information input. In terms of inspection standard entry, it is released, updated and deleted according to national standards. It is highlighted when updated, so as to remind users to query and use. In the input of inspection information, the food inspection data provided by the inspection institution shall be recorded in sequence. This function provides an import function to reduce the workload of the staff.

Table 1 Risk factor information

\begin{tabular}{cccc}
\hline Name & Type & Scale & Emptiness \\
\hline Risk ID & int & & no \\
Riskname & nvarchar & 50 & no \\
Risklevel & Int & & No \\
Specialised & Nvarchar & 100 & No \\
Object & Nvarchar & 100 & No \\
System & Nvarchar & 100 & No \\
Subsystem & nvarchar & 100 & no \\
\hline
\end{tabular}

The early warning system consists of a user input interface, a data input interface, a risk data mining module, a risk factor library, an early warning function module, and an output interface.

For management-type automatic alarms, historical data and monitoring data are input to the 
background of the system through the data input interface. Data mining in the risk factor database is performed by the risk data mining module. The demand information is passed through the early warning function module, and the warning information is output to the client interface. On the management of artificial alarm: on-site workers or security management personnel through the inspection, the risk information of the production site, through the client interface, the risk information is input to the background of the system, through the risk data mining module in the risk factor database data mining The demand information is passed through the early warning function module and the warning information is output to the client interface.

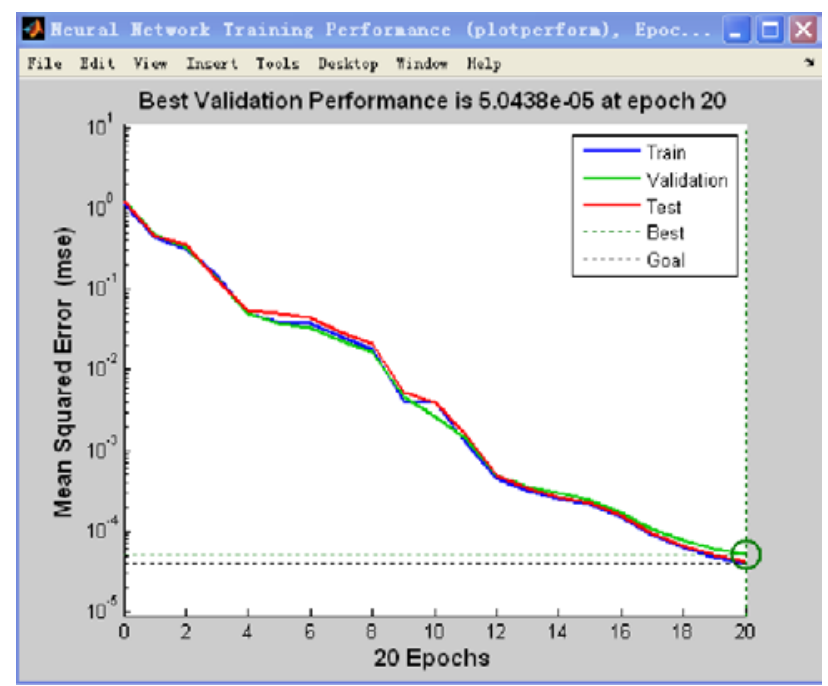

Fig. 3 Training process

The early warning rule set is extracted according to the attributes of the input data, and then a matching analysis is performed for each rule. When matching early warning, the first item of the current rule is found in the risk factor library. If it can not be found, it is considered that the rule does not apply to the record, abandoning the match and turning to the next rule of the rule set; if found, the rule is considered to be applicable to the record, and the early warning module is based on the pre set early warning strategy. Comprehensive matching results are used to generate early warning information.

\section{Summary}

This paper proposes the application of MATLAB2012a neural network tool to the community risk coefficient data mining, the construction of community safety risk warning system. To select appropriate training methods through a large number of community risk factors as input, then use the data to verify it, and update the database in a timely manner to use new data for training in forecasting systems, The community safety warning method based on BP neural network data mining can predict the input batch effectively. The method is simple to operate, which is very helpful to the grassroots community safety supervision department to improve the relevant early warning techniques, support the supervision department to clarify the regulatory priorities at each time, and eventually improve the effectiveness of the regulatory decision-making, so that the limited resources can produce the maximum regulatory effect.

\section{References}

[1] Koyuncugil A S, Koyuncugil A S, Ozgulbas N: Surveillance Technologies and Early Warning Systems (Data Mining Applications for Risk Detection, 2011) p. 59-63.

[2] Ozgulbas N, Koyuncugil A S: Risk Classification of SMEs by Early Warning Model Based on Data Mining (World Academy of Science Engineering \& Technology, 2012), p.83-89. 
[3] Koyuncugil A S, Ozgulbas N: Financial early warning system model and data mining application for risk detection (Expert Systems with Applications, 2012), 39(6), p.6238-6253.

[4] Luo Y M: Research on Financial Risk Analysis and Early Warning Based on Data Mining Technology (Computer Knowledge \& Technology, 2015) p.93-99. 